\title{
ANÁLISE COMPARATIVA DOS ENSAIOS ACELERADOS DE EXPANSÃO DE BARRAS DE ARGAMASSA DE CIMENTO PORTLAND NO ATAQUE EXTERNO POR SULFATOS DE SÓDIO: NBR 13583/2014 E ASTM C 1012/2018
}

\author{
TOZETTO, ANA ELISA \\ Engenheira Civil \\ Universidade Estadual de Ponta Grossa \\ Paraná; Brasil \\ anaelisatozetto@gmail.com
}

\section{PEREIRA, EDUARDO}

Professor Doutor

Universidade Estadual de Ponta Grossa

Paraná; Brasil

eduardopereira@uepg.br

\author{
BREKAILO, FERNANDA \\ Professora Mestre \\ Universidade Estadual de Ponta Grossa \\ Paraná; Brasil \\ fernandabrekailo@gmail.com
}

\section{RESUMO}

A NBR 13583/2014 estabelece o método de ensaio para determinação da variação dimensional de barras de argamassa de cimento Portland expostas a solução de sulfato de sódio e a NBR 16697/2018 apresenta o limite de expansão para que o cimento seja considerado resistente a sulfatos. $O$ interesse por este estudo surge em razão da necessidade de se ter parâmetros de expansão nas mais variadas situações, utilizando diferentes tipos de materiais e inserido nos diversos meios. Por este motivo, utilizou-se a normativa americana ASTM C 1012/2018 em conjunto com o Guide to Durable Concrete do ACI, que especifica a composição da matriz cimentícia para o meio ambiente que o concreto estará inserido e limita a expansão linear para os diferentes tipos de cimento, no caso do ataque por sulfatos de origem externa. Ao analisar ambas as normas, verificam-se alguns pontos divergentes no procedimento experimental, como o traço da argamassa, temperatura de exposição ao agente agressivo e a concentração da solução agressiva. Realizou-se então ensaios de determinação de variação dimensional de barras de argamassa utilizando a metodologia de ambas normativas. Os resultados evidenciam que para as condições da ASTM, os traços apresentam expansões inferiores aos limites recomendados para esta norma. Ao aumentar-se a concentração da solução, os traços apresentam expansões superiores aos limites. Confirma-se pela metodologia brasileira que ambos traços são sensíveis aos sulfatos. Assim, a metodologia da ASTM não foi adequada para os materiais utilizados aqui no Brasil, ou os limites indicados são muito brandos para o tempo de análise.

Palavras-chave: NBR 13583/2014, ASTM C 1012/2018, Ataque externo por sulfato, Expansão de argamassas.

\begin{abstract}
The NBR 13583/2014 defines the test method that determinates the dimensional variation of Portland cement mortar bars exposed to sodium sulfate solution, while the NBR 16697/2018 defines the limit expansion for the cement to be considered sulfate resistant. There is a need for parameters of expansion in different situations, using different types of materials and environments, manifesting the interest for this type of study. For this reason, the American standard ASTM C 1012/2018 was used with the Guide to Durable Concrete of ACI, which one specifies the composition of the cementitious matrix for the environment where the concrete will be inserted and define limits for the linear expansion of the different types of cement, in cases of external sulfate attack. When analyzing both standards, there are some divergent points in the experimental procedure, such as the mix of the mortar, the exposure temperature to the aggressive agent and the aggressive solution concentration. Therefore, the tests of dimensional variation determination of mortar bars were performed using the methodology of both standards. The results showed that for ASTM conditions, the mixes had expansions below the limits recommended for this standard. By increasing the concentration of the solution, the mixes had expansions above the limits. It is confirmed by the Brazilian methodology that both mixes are sensitive to sulfates. Thus, the ASTM methodology is not suitable for the materials used in Brazil, or the indicated limits are too low for the analysis time.
\end{abstract}

Keywords: NBR 13583/2014, ASTM C 1012/2018, External sulfate attack, Expansion of mortars. 


\section{INTRODUÇÃO}

$\mathrm{O}$ ataque por sulfatos é uma das manifestações patológicas que podem ocorrer nas matrizes cimentícias de estruturas de concreto. Este tipo de degradação é caracterizado pela reação entre íons sulfatos $\left(\mathrm{SO}_{4}{ }^{2-}\right)$ e os componentes formados

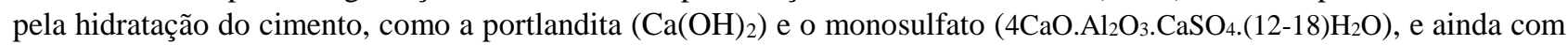
o $\mathrm{C}_{3} \mathrm{~A}$ anidro presente na matriz. Os principais produtos formados por estas reações são cristais de etringita $\left(6 \mathrm{CaO} . \mathrm{Al}_{2} \mathrm{O}_{3} \cdot 3 \mathrm{CaSO}_{4} \cdot 32 \mathrm{H}_{2} \mathrm{O}\right)$ e gipsita $\left(\mathrm{CaSO}_{4} \cdot 2 \mathrm{H}_{2} \mathrm{O}\right)$. Essas reações podem ocorrer tanto pela ação de sulfatos de origem interna (ataque interno por sulfatos) quanto de origem externa (ataque externo por sulfatos ou ataque ácido) e tem como resultado o desenvolvimento de um processo expansivo. O processo é considerado expansivo pois os cristais de etringita e gipsita formados ocupam um volume maior que os componentes que lhe deram origem. As expansões podem ter como consequência um progressivo processo de fissuração, podendo levar o concreto ao colapso (SANTHANAM; COHEN; OLEK, 2003; MEHTA; MONTEIRO, 2006).

Ao ser exposto a meios com presença de sulfatos, o tempo para o desenvolvimento da deterioração do concreto e a agressividade com que este fenômeno ocorrerá, irá depender de fatores como a dosagem do compósito cimentício, do tipo de cimento utilizado, da temperatura a que está exposto e a concentração da solução no meio. $O$ avanço das reações geralmente é de difícil identificação nas primeiras idades. Quando da continuidade das reações, originam-se tensões internas na matriz, que levam a expansão, podendo gerar fissuras. A propagação destas fissuras aumentam a permeabilidade do concreto, promovendo assim, condições propícias para a evolução das reações ou ainda para entrada de outros agentes agressivos (SKALNY; MARCHAND; ODLER, 2002; PEREIRA, 2015).

Dessa maneira, buscam-se meios para compreender os estágios de reação, os produtos formados, a interação dos materiais e formas de resistir aos ataques por sulfatos. A normatização no Brasil é feita pela NBR 13583 (ABNT, 2014) para avaliação da resistência de argamassas a este tipo de degradação, baseando-se no ensaio de medição da expansão linear percentual de barras de argamassa, em condições especificadas pela referida norma. De maneira similar a norma brasileira, tem-se a norma americana ASTM C 1012 (2018), que também traz o procedimento de ensaio de expansão das barras de argamassa expostas a solução de sulfatos. Em conjunto com a normativa, o Guide to Durable Concrete (ACI, 2001) especifica a composição da matriz cimentícia para o meio ambiente que o concreto estará inserido e limita a expansão linear para os diferentes tipos de cimento, no caso do ataque por sulfatos de origem externa.

Mesmo o ataque por sulfatos de origem externa sendo bastante conhecido e já existirem normativas no Brasil para avaliação dos aglomerantes frente a esta manifestação patológica, não existiam parâmetros normativos quanto a expansão máxima permitida para uma argamassa ser considerada resistente a sulfatos. Alguns trabalhos científicos no Brasil, como Hoppe Filho et al. (2015), Souza (2006) e Souza (2016), utilizavam como referência o valor de 0,03\% de expansão aos 56 dias, sendo 14 dias de cura das amostras seguidos por 42 dias em exposição à solução, conforme sugerido por Marciano (1993). Recentemente, uma nova atualização da norma NBR 16697 (ABNT, 2018) - Cimento Portland: Requisitos, inseriu este valor como limite de expansão, percebendo-se a necessidade em uniformizar os dados obtidos através dos ensaios de ataque por sulfatos, bem como analisar a máxima expansão que a estrutura poderá estar exposta.

Apesar dos avanços nas legislações, as atuais referências para comparação dos ataques por sulfatos ainda apresentam lacunas que demandam estudos. Na NBR 13583 (ABNT, 2014), verifica-se falta de dados e detalhamento de procedimentos, enquanto na ASTM C 1012 (2018), os tempos para execução dos ensaios e posterior análise são demasiadamente longos, podendo chegar a 18 meses (SOUZA, 2006). A falta de dados da norma brasileira é em relação a execução do ensaio, como quantidade de golpes para adensamento da argamassa nos moldes, aos diferentes tipos de cimento que podem ser utilizados em locais com possível exposição ao sulfato, mas principalmente, uma análise dos resultados, com valores máximos de expansão para os diferentes tipos de cimento que podem ser utilizados em diferentes classes de exposição, e não apenas para o cimento específico resistente aos sulfatos. Os valores máximos de expansão para cada tipo de cimento classificados conforme o ambiente inserido é de suma importância, pois nem sempre será possível, viável ou até necessário utilizar o cimento especificado resistente a sulfatos (RS), dependendo da concentração iônica presente.

Por outro lado, baseado nas experiencias e pesquisas daquele país, a normativa americana propõe porcentagens máximas de expansão para cada tipo de cimento utilizado e qual o tempo necessário de análise conforme cada um. Assim, o intuito deste trabalho é fazer um apanhado das duas normas e verificar a influência de cada variável para a expansão das barras de argamassa, realizando o estudo comparativo das metodologias e associações entre os limites de expansão propostos. 
De acordo com Souza (2016), apesar da norma NBR 13583 (ABNT, 2014) apresentar um ensaio acelerado de expansão, os 42 dias propostos podem não ser um tempo de ensaio satisfatório para determinar o grau de susceptibilidade das barras de argamassa ao ataque por sulfatos. A justificativa do autor é que para cimentos compostos, como CP II Z, que contém adição pozolânica, a expansão pode ocorrer em um tempo mais longo de exposição, tendo em vista que a pozolana apresenta o efeito mitigador e assim, as reações demoram mais para se desenvolverem.

Dessa maneira, o estudo das variáveis envolvidas em cada procedimento torna-se um fator importante para análise dos resultados a fim de não levar a erros de interpretação da susceptibilidade das estruturas ao ataque por sulfatos, conforme o meio inserido e os materiais utilizados. Assim, o objetivo desta pesquisa foi realizar uma análise comparativa do comportamento de barras de argamassa de cimento Portland submetidas aos procedimentos propostos na NBR 13583 (ABNT, 2014) e ASTM C 1012 (2018) para avaliação do ataque externo por sulfatos, avaliando a influência dos parâmetros de ensaio que diferem entre as normativas nas alterações microestruturais da argamassa bem como os limites de expansão propostos pelas normas.

\section{MATERIAIS E MÉTODOS}

\subsection{Materiais}

O aglomerante utilizado para moldagem das barras de argamassa para o ensaio de ataque por sulfatos foi o cimento Portland CP II-F 32. Para caracterização química dos cimentos foi utilizado o ensaio de fluorescência de raios X (FRX), realizado em espectrômetro modelo Rigaku ZSX Primus II. Na Tabela 1 tem-se as características químicas do cimento utilizado, e todos os índices apresentam valores dentro dos limites especificados pela NBR 16697 (ABNT, 2018).

Tabela 1 - Caracterização físico-química do cimento CP II-F 32.

\begin{tabular}{cccccccc}
\hline $\mathrm{CaO}$ & $\mathrm{SiO}_{2}$ & $\mathrm{MgO}$ & $\mathrm{Al}_{2} \mathrm{O}_{3}$ & $\mathrm{SO}_{3}$ & $\mathrm{Fe}_{2} \mathrm{O}_{3}$ & $\mathrm{~K}_{2} \mathrm{O}$ & Perda ao fogo \\
\hline 59,2 & 13,6 & 2,32 & 3,30 & 3,11 & 2,50 & 0,834 & 10,64 \\
\hline
\end{tabular}

A areia utilizada é proveniente de leito de rio, com granulometria fina e mineralogia quartzosa. O material foi caracterizado quanto a distribuição granulométrica pelas normas NBR 7217 (ABNT, 1987) e NBR 7211 (ABNT, 2005), apresentando distribuição na zona utilizável inferior. A solução agressiva utilizada para os ensaios foi preparada com sulfato de sódio anidro $\left(\mathrm{Na}_{2} \mathrm{SO}_{4}\right)$.

\subsection{Ensaios realizados}

2.2.1 Determinação da variação dimensional de barras de argamassa expostas ao ataque externo por sulfatos

O ensaio para determinação da variação dimensional das barras de argamassa foi realizado conforme proposto na NBR 13583 (ABNT, 2014) e ASTM C 1012 (2018). Moldaram-se 8 barras seguindo o procedimento de mistura conforme NBR 7215 (ABNT, 1996), adotando-se duas camadas de preenchimento dos moldes com 20 golpes com soquete cada. A cura inicial foi feita ao ar por 2 dias na câmara úmida, e então desmoldados para imersão na solução saturada com cal por 12 dias, para cura intermediária. Após o período de cura intermediário, realizou-se a leitura inicial do comprimento de cada barra por meio um pórtico com relógio digital comparador.

As amostras são então submetidas à cura final, que corresponde ao período de exposição às soluções de análise, onde quatro barras de cada grupo é imersa em solução de sulfato de sódio e as demais em solução de água saturada com cal. Realizaram-se medidas semanais das dimensões das barras com o relógio comparador. A determinação da expansão relativa é dada pela diferença entre a variação linear das barras imersas em solução de sulfato com as barras de mesmo traço imersas em solução de água saturada com cal.

Adotou-se os traços apresentados por ambas normativas para as diferentes situações de exposição analisadas. O traço apresentado pela NBR 13583 (ABNT, 2014) é de 1:3,2 com fator água/cimento de 0,60, enquanto o apresentado pela ASTM C 1012 (2018) é de 1:2,75 com a/c de 0,485.

Os ensaios procederam em duas frentes de análise, a primeira levando-se em consideração a execução conforme a normativa americana, com o tempo de ensaio requerido à temperatura ambiente, e a segunda, conforme a normativa brasileira, realizado em estufa. O primeiro grupo de amostras foi exposto à solução de sulfato na temperatura de $23 \pm$ $2^{\circ} \mathrm{C}$, em câmara úmida durante 6 meses de exposição à soluções com concentração de 5 e $10 \%$ de sulfato de sódio. $\mathrm{O}$ 
segundo grupo foi exposto exposto à solução de sulfato na temperatura de $40 \pm 2^{\circ} \mathrm{C}$, em estufa durante 42 dias de exposição à soluções com concentração de $10 \%$ de sulfato de sódio.

\subsubsection{Análises microestruturais}

Após o fim da cura final, de imersão na solução agressiva, foram preparadas amostras dos traços para análise no microscópio eletrônico de varredura com EDS. As imagens em microscopia foram obtidas com o uso de um microscópio FEG SEM, Tescan, Mira 3, com detectores SE, BSE e microssonda analítica de raios X Oxford X-Maxn 50 (EDS), com metalização superficial das amostras em ouro, com o intuito de analisar a formação dos cristais na microestrutura das argamassas após o ataque por sulfatos.

\section{RESULTADOS E DISCUSSÕES}

Os dados obtidos de expansão das barras de argamassa até a idade de 6 meses em solução com concentração de 5 e $10 \%$ de sulfato de sódio, para as argamassas mantidas em temperatura ambiente, estão apresentados na Figura 1.

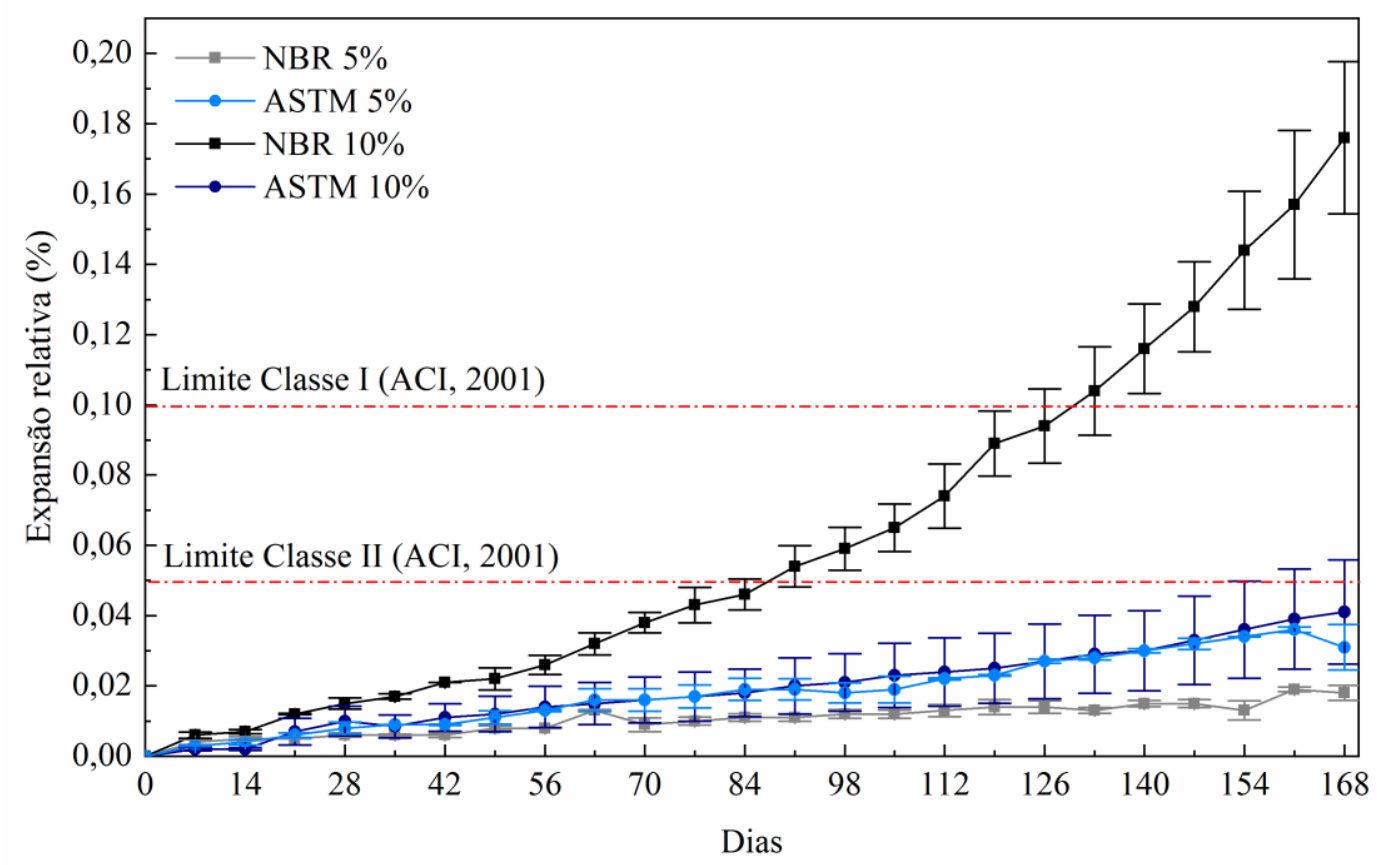

Figura 1: Expansão relativa das barras de argamassa imersas em solução de 5 e $10 \%$ de $\mathrm{Na}_{2} \mathrm{SO}_{4}$, em temperatura ambiente

As expansões de cada grupo de amostras foram comparadas com limites apresentados pelo Guide to Durable Concrete do ACI (ACI, 2001), para ensaios realizados em 6 meses de exposição em ambientes classificados como classe I e II de agressividade, podendo ter expansão máxima de $0,10 \%$ e $0,05 \%$ respectivamente. Assim, nenhuma amostra obteve expansões maiores que os limites durante o desenvolvimento do ensaio com a concentração de $5 \%$ de sulfato de sódio. Já para as concentrações de $10 \%$ de sulfato de sódio, ambos traços apresentam expansões superiores aos limites para classe I e II após 6 meses de análise.

Nota-se que as argamassas com o traço da normativa brasileira apresentaram maior sensibilidade a mudança de concentração da solução, apresentando expansão superior para a maior concentração. Já as barras de argamassa com traço da normativa americana apresentaram expansões similares para ambas concentrações. O maior fator água/cimento da normativa brasileira pode ter tornado a argamassa menos resistente e mais porosa, facilitando a entrada da solução agressiva. Desta forma, ao aumentar a concentração de sulfato na solução, elevou-se a quantidade de íons sulfatos na matriz, passíveis de reagir com os componentes da pasta hidratada. O comportamento apresentado está de acordo com o esperado ao elevar-se a concentração da solução, conforme apresentam Santhanam, Cohen e Olek (2002). 
O traço da norma americana apresentou pequena sensibilidade a mudança de concentração, considerando-se que a amostra imersa em solução de $10 \%$ atingiu o limite de expansão apenas pela análise da barra de erros. O cimento adotado nesta pesquisa (CP II-F 32) não é um cimento considerado resistente à sulfatos pela normativa brasileira, sendo assim, o resultado com a concentração de $5 \%$ e traço sugerido pela norma americano não é correspondente ao esperado para este tipo de cimento.

Ao analisar os traços para situação de exposição em estufa à $40^{\circ} \mathrm{C}$, obteve-se as expansões resultantes apresentadas na Figura 2.

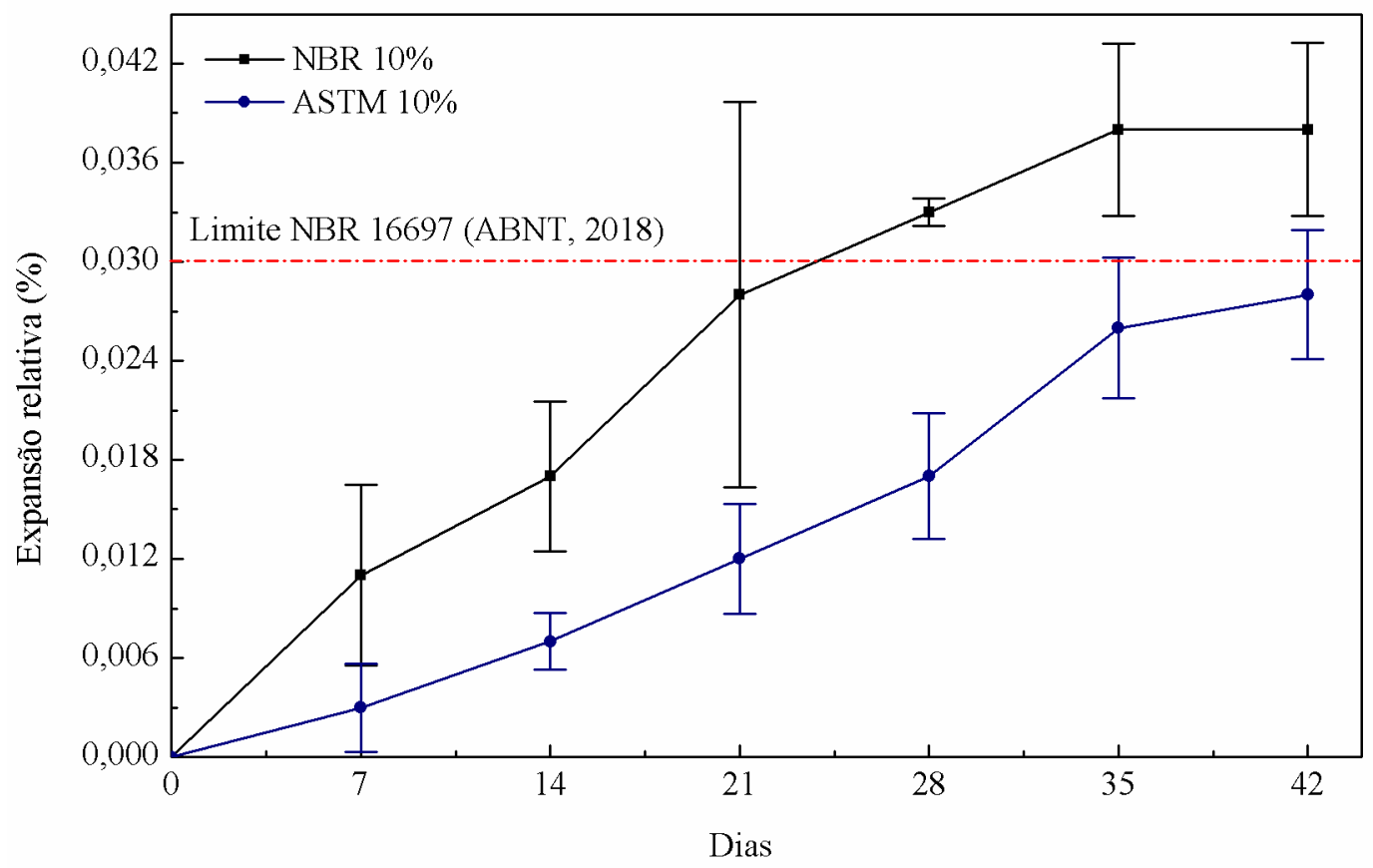

Figura 2: Expansão relativa das barras de argamassa imersas em solução de $10 \%$ de $\mathrm{Na}_{2} \mathrm{SO}_{4}$, em estufa à $40^{\circ} \mathrm{C}$

Na comparação para a normativa brasileira aos 42 dias de exposição, o limite apresentado pela NBR 16697 (ABNT, 2018) é de $0,03 \%$ de expansão relativa. Neste caso, ambas amostras apresentaram expansões superiores ao limite, sendo que o traço da normativa americana novamente ultrapassa o limite pela análise da barra de erros das expansões. A falta de dados de expansão para ambos traços ou demais variáveis das metodologias para o mesmo tipo de cimento impede a comparação destes dados com a literatura.

Analisando as imagens da microestrutura das argamassas, a amostra com o traço da NBR imersa em solução de 5\% de sulfato de sódio apresenta os poros preenchidos por cristais aciculares de etringita (Figura 3a). Na amostra com traço de ASTM, também exposta à solução de 5\%, também é possível observar a formação aglomerada de cristais de etringita (Figura 3b). Para a solução de $10 \%$, a amostra com traço na NBR também apresenta grande quantidade cristais de etringita preenchendo os poros (Figura 3c). Já para o traço da ASTM nesta solução, observa-se uma alteração na morfologia dos cristais que levam a uma alteração da estrutura interna dos poros (Figura 3d). Acredita-se que a intensidade do ataque alterou a estrutura interna dos poros e que neste caso a etringita não é o produto prioritário encontrado, ao contrário do esperado, devendo a expansão do ataque estar associado a estas alterações. As imagens estão de acordo com o observado para as expansões das barras com o traço da ASTM, onde o aumento na concentração da solução não resultou em elevada mudança na intensidade do ataque. 
a) NBR $5 \%$

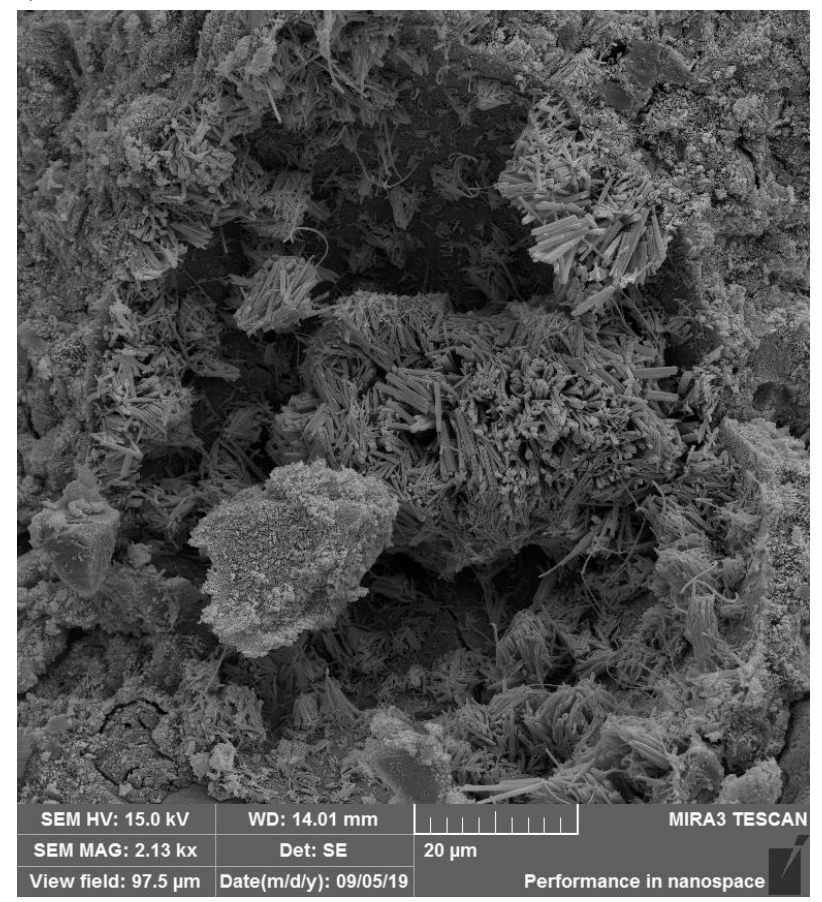

c) NBR $10 \%$

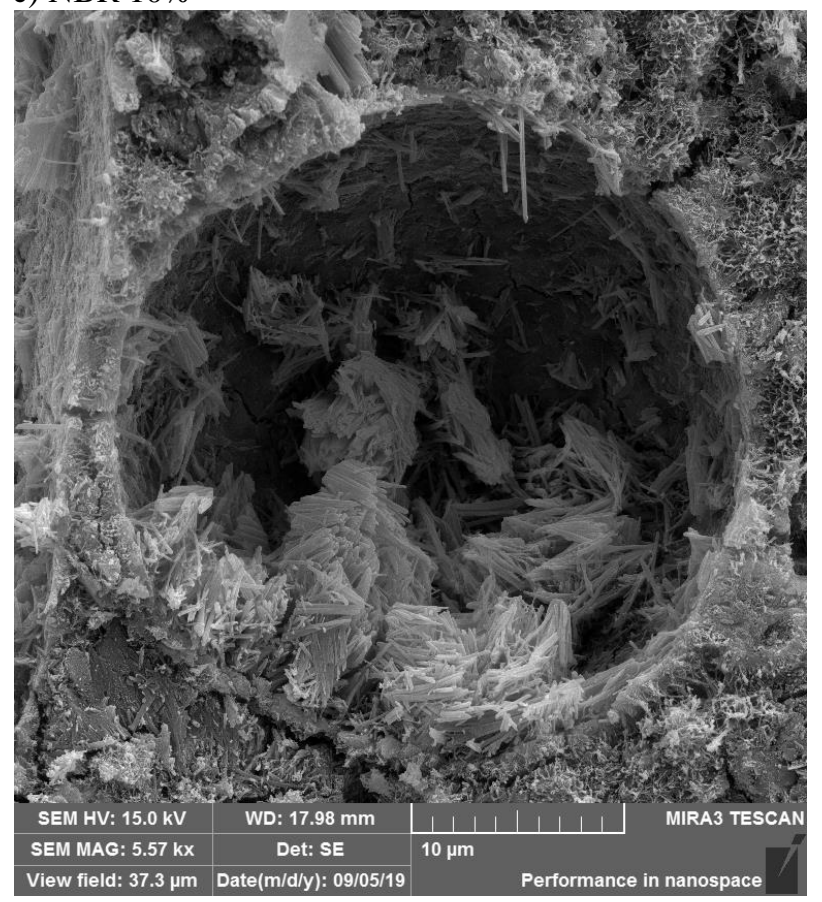

b) ASTM 5\%

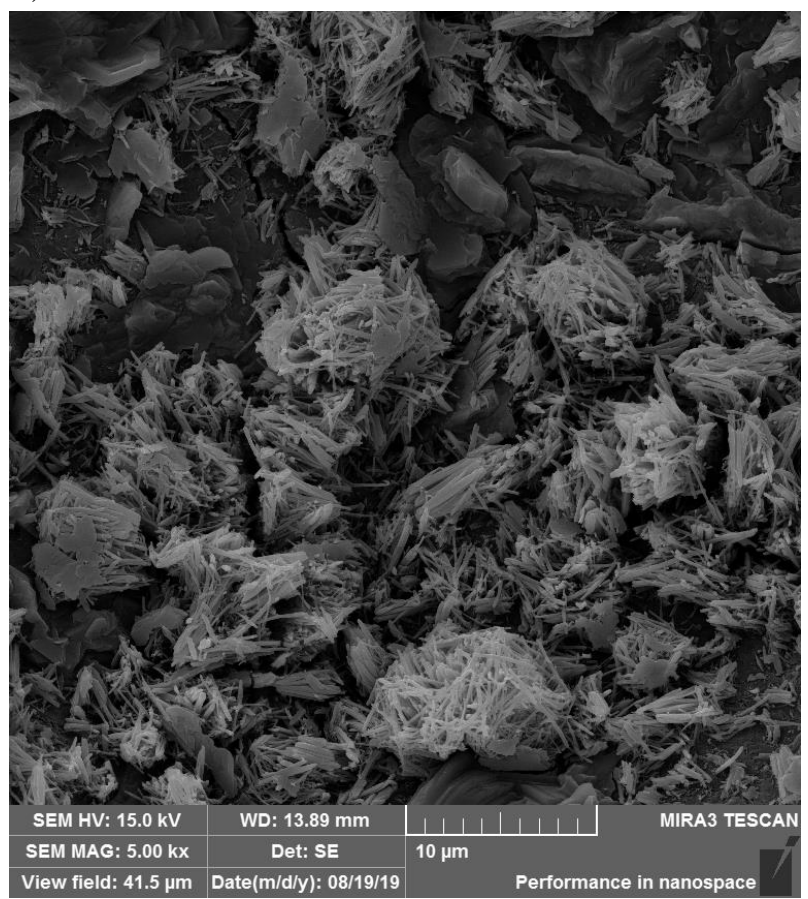

d) ASTM $10 \%$

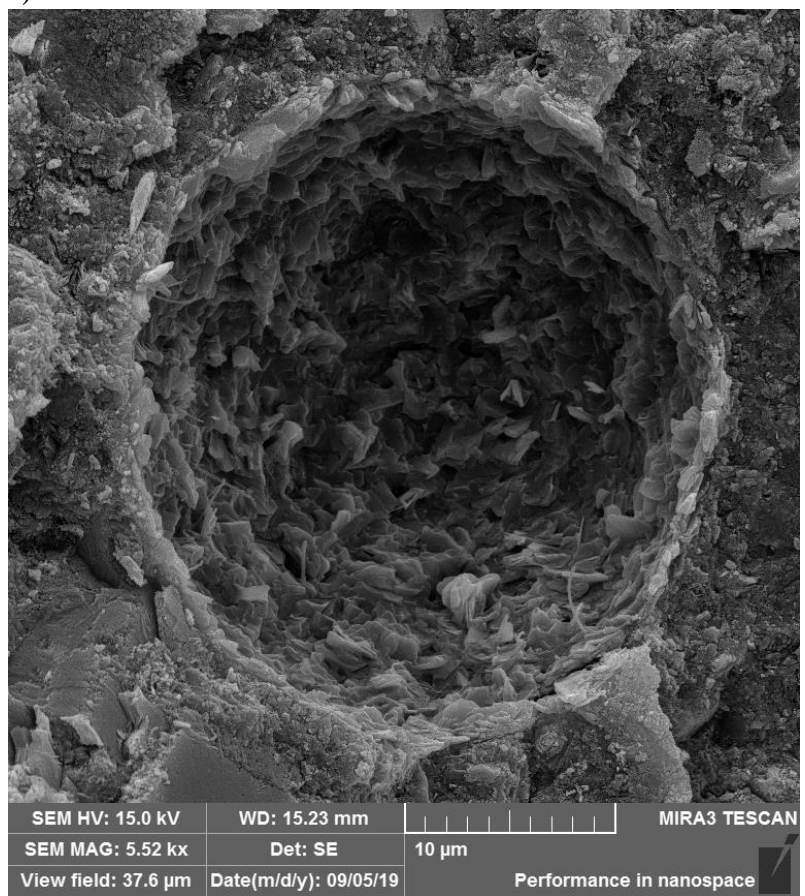

Figura 3: Microestrutura das argamassas após imersão em solução de sulfatos por 6 meses em temperatura ambiente: (a) NBR 5\%; (b) ASTM 5\%; (c) NBR 10\%; (d) ASTM 10\%

Para as amostras em que o ensaio foi realizado em estufa, é possível verificar a influência da temperatura na velocidade das reações, principalmente para o traço da norma americana, onde a argamassa apresentou poros completamente tamponados por cristais de etringita (Figura 4b). Para o traço da norma brasileira, note-se o alinhamento dos cristais formados perpendicularmente aos poros (Figura 4a). 
a) NBR $10 \%$

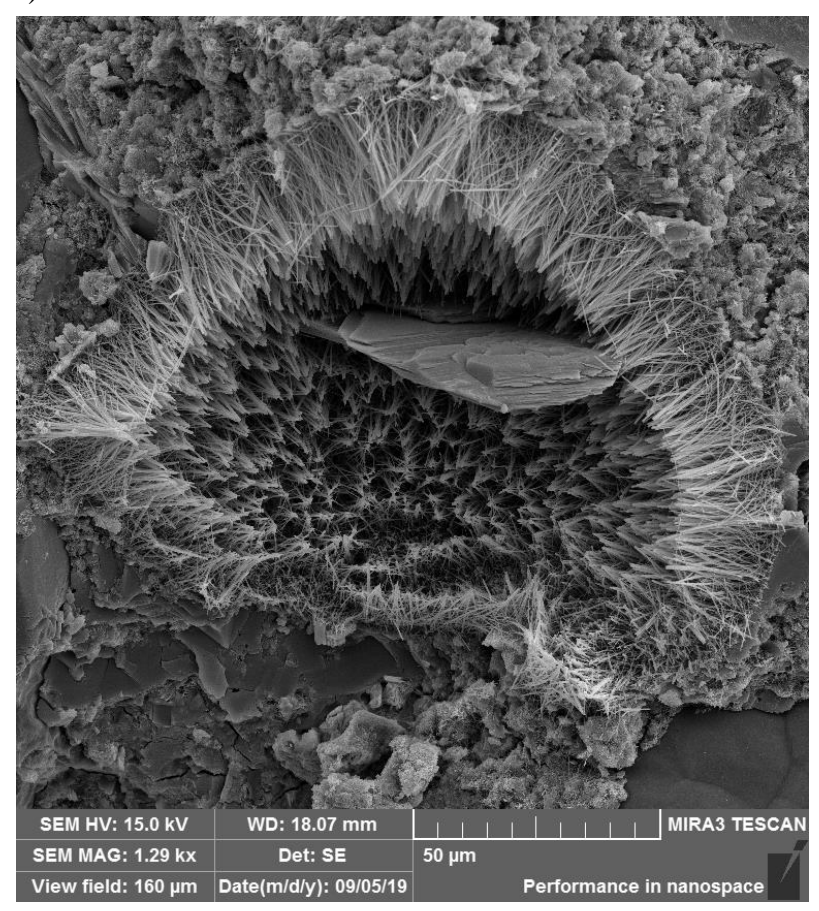

b) ASTM $10 \%$

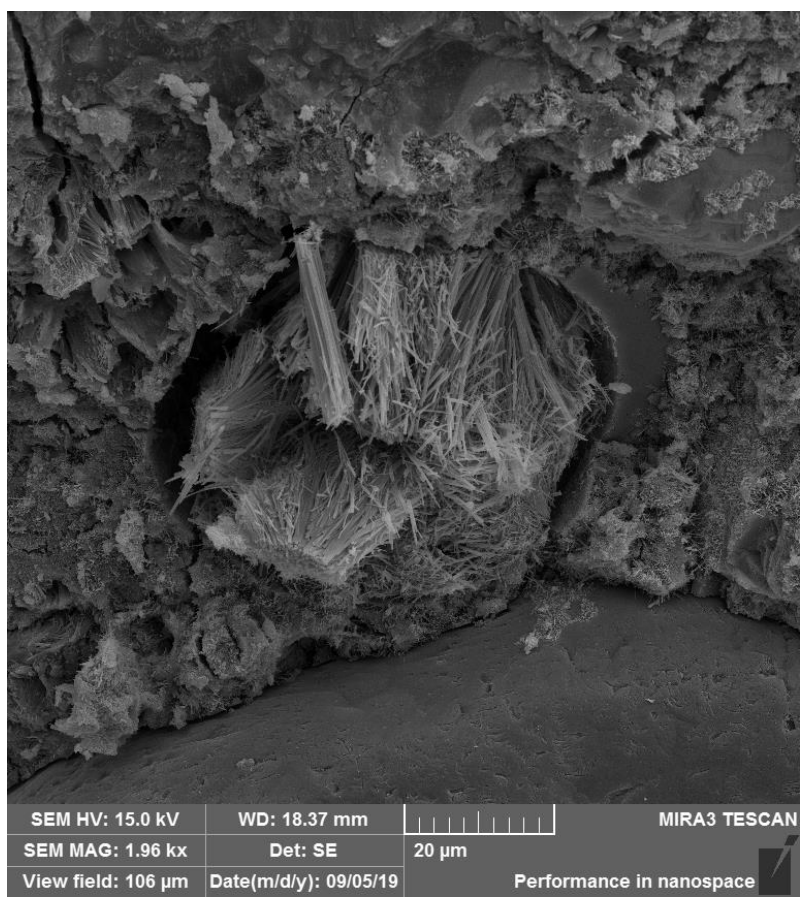

Figura 4: Microestrutura das argamassas após imersão em solução de sulfatos por 42 dias em estufa à $40^{\circ} \mathrm{C}$ :

(a) NBR 10\%; (b) ASTM 10\%

\section{CONCLUSÕES}

Este trabalho apresentou como principal objetivo a comparação de desenvolvimento do ensaio de determinação da expansão relativa de barras de argamassa imersas em solução agressiva de sulfato de sódio entre a normativas brasileira NBR 13583 (ABNT, 2014) e americana ASTM C 1012 (2018).

O cimento brasileiro, apesar de pureza menor do que os cimentos americanos (em termos de teor de clínquer), nas condições propostas pela ASTM C 1012 (2018), com exposição em temperatura ambiente e concentração de solução de $5 \%$, não ultrapassou os limites estabelecidos para ensaios desenvolvidos em 6 meses de ensaio, tanto para o traço da norma americana como da brasileira. O limite foi ultrapassado em temperatura ambiente quando aumentou-se a concentração da solução para 10\%. Verificou-se que o maior fator água/cimento, indicado pela normativa brasileira, torna as argamassas mais sensíveis as mudanças de concentração, possivelmente pela redução na resistência e aumento da porosidade. $\mathrm{O}$ ensaio realizado conforme metodologia da norma brasileira, durante 42 dias em temperatura de $40^{\circ} \mathrm{C}$, confirma que ambos traços ultrapassam os limites de expansão para a referida idade, não sendo resistentes ao ataque externo por sulfatos.

Verifica-se que a metodologia desenvolvida pela ASTM C 1012 (2018) não foi adequada para os materiais utilizados no Brasil, ou os limites indicados são muito brandos, pois sabe-se que o cimento CP II-F não tem resistência ao ataque por sulfatos, comprovando a importância de uma dosagem adequada e fator água/cimento compatível com os materiais empregados. Destaca-se que para um ensaio de curto período, a metodologia proposta pela normativa brasileira pode ser eficaz, porém, recomenda-se ensaios mais longos para maior acompanhamento das reações e análise dos ambientes de exposição, a fim de obter-se limites para diferentes situações de exposição.

\section{AGRADECIMENTOS}

Os autores agradecem a Universidade Estadual de Ponta Grossa por fornecer a infraestrutura para esta pesquisa, em particular ao Complexo de Laboratórios Multiusuários (C-LABMU), e a Fundação Araucária pelo financiamento na forma de bolsas de estudo e equipamentos. 


\section{REFERÊNCIAS}

AMERICAN CONCRETE INSTITUTE. ACI 201.2R-01: Guide to Durable Concrete. Estados Unidos, 2000.

ASTM INTERNATIONAL. ASTM C 1012: Standard Test Method for Length Change of Hydraulic-Cement Mortars Exposed to a Sulfate Solution. Estados Unidos, 2018.

ASSOCIAÇÃO BRASILEIRA DE NORMAS TÉCNICAS. NBR 7211: Agregados para concreto - Especificação. Rio de Janeiro, 2005.

ASSOCIAÇÃO BRASILEIRA DE NORMAS TÉCNICAS. NBR 7215: Cimento Portland - Determinação da resistência à compressão de corpos de prova cilíndricos. Rio de Janeiro, 1996.

ASSOCIAÇÃO BRASILEIRA DE NORMAS TÉCNICAS. NBR 7217: Agregados - Determinação da composição granulométrica. Rio de Janeiro, 1987.

ASSOCIAÇÃO BRASILEIRA DE NORMAS TÉCNICAS. NBR 13583: Cimento Portland - Determinação da variação dimensional de barras de argamassa de cimento Portland expostas à solução de sulfato de sódio. Rio de Janeiro, 2014.

ASSOCIAÇÃO BRASILEIRA DE NORMAS TÉCNICAS. NBR 16697: Cimento Portland - Requisitos. Rio de Janeiro, 2018.

HOPPE FILHO, J.; SOUZA, D. J.; MEDEIROS, M. H. F. de; PEREIRA, E.; PORTELA, K. F. Ataque de matrizes cimentícias por sulfato de sódio: adições minerais como agentes mitigadores. Cerâmica 61, 168-177. 2015.

MARCIANO, Z. A. N. Desenvolvimento de um método acelerado para avaliação da resistência de argamassas de cimento Portland expostas à Solução de Sulfato de Sódio. 1993. 202f. Dissertação (Mestrado) - Escola Politécnica da Universidade de São Paulo. São Paulo, 1993.

MEHTA, P. K.; MONTEIRO, P. J. M. Concrete: microstructure, properties and materials. $3^{\text {a }}$ Edição. Estados Unidos: The McGraw-Hill Companies, 2006. 684p.

PEREIRA, E. Investigação e monitoramento do ataque por sulfatos de origem interna em concretos nas primeiras idades. Tese (Doutorado em Engenharia e Ciência dos Materiais) - Universidade Federal do Paraná. Curitiba, 2015.

SANTHANAM, M.; COHEN, M. D.; OLEK J. Mechanism of sulfate attack: A fresh look - Part 2: Proposed mechanisms. Cement and Concrete Research, n.33, p.341-346, 2003.

SANTHANAM, M.; COHEN, M. D.; OLEK J. Modeling the effects of solution temperature and concentration during sulfate attack on cement mortars. Cement and Concrete Research, n.32, p.585-592, 2002.

SKALNY, J.; MARCHAND, J.; ODLER, I. Sulfate attack on concrete. New York. Spon spress. 2002.

SOUZA, D.J. Capacidade de adições minerais em mitigar o ataque por sulfatos de sódio e magnésio em argamassas de cimento Portland. Dissertação (Mestrado em Engenharia de Construção Civil) - Universidade Federal do Paraná. Curitiba, 2016.

SOUZA, R. B. Suscetibilidade de Pastas de Cimento ao Ataque por Sulfatos - método de ensaio acelerado. $131 \mathrm{f}$. Dissertação (Mestrado em Engenharia) - Universidade de São Paulo. São Paulo, 2006. 